\title{
Oncological Outcomes of Non-Urothelial Bladder Cancers in a Specialized Cancer Hospital of a Developing Country
}

Siddique Adnan 1, 2 , Muhammad Arshad Irshad Khali1 ${ }^{1}$, Shaukat Fiaz ${ }^{1}$, Muhammad Abu Bakar ${ }^{3}$, Azfar Al ${ }^{1}$, Zubair Ahmad Cheema ${ }^{1}$, Khurram Mir ${ }^{1}$

1. Surgical Oncology, Shaukat Khanum Memorial Cancer Hospital and Research Centre, Lahore, PAK 2. Urology, Institute of Kidney Diseases, Peshawar, PAK 3. Biostatistics and Epidemiology, Shaukat Khanum Memorial Cancer Hospital and Research Centre, Lahore, PAK

Corresponding author: Siddique Adnan, siddiquermi@gmail.com

\section{Abstract \\ Background}

Non-urothelial bladder cancers (NUBCs) constitute only $5 \%$ of all bladder cancers. Because of the scarcity of data, no standardized treatment can be offered to these patients. Surgical treatment can be offered to patients with localized disease; however, generally, the prognosis is unfavorable.

\section{Methodology}

Patients with histology-proven NUBC presenting to the Department of Uro-oncology, Shaukat Khanum Memorial Cancer Hospital and Research Center, Lahore, Pakistan, from January 2002 to July 2017 were identified and assessed retrospectively.

\section{Results}

A total of 20 patients with a median presenting age of 52 years (range: 34-87 years) were identified. Clinically, T3 was the commonest stage of presentation, i.e., in 11 (55\%), whereas 1 (7.1\%) patient had metastatic disease. Four types of NUBCs were identified: adenocarcinoma, squamous cell carcinoma, small cell carcinoma, and inflammatory myofibroblastic tumor. Most of the patients with adenocarcinoma were offered surgical treatment in the form of either partial (64.3\%) or radical (28.6\%) cystectomy. Two patients with small cell carcinoma and two of the three patients with SCC could only be offered palliative chemotherapy. During a median follow-up of 40 months, 14 (70\%) patients developed disease progression or recurrence. All these patients succumbed to their disease during a median period of 37.5 months (range: 584 months). Furthermore, three- and five-year disease-free survival was $60 \%$ and $51 \%$, respectively, and overall survival was $65 \%$ and $31 \%$, respectively.

Received 07/28/2020

Review began 08/10/2020 Review ended 08/14/2020 Published 08/23/2020

\section{() Copyright 2020}

Adnan et al. This is an open access article distributed under the terms of the Creative Commons Attribution License CC-BY 4.0., which permits unrestricted use, distribution, and reproduction in any medium, provided the original author and source are credited.

\section{Conclusions}

NUBC is a rare but aggressive disease that presents at an advanced stage in many cases. Treatment protocols are not uniform; therefore, further collaborative research is needed to improve survival outcomes.

\section{Categories: Urology, Oncology, Other}

Keywords: non-urothelial bladder cancer, radical cystectomy, chemotherapy

\section{Introduction}

Bladder cancer (BC) is the 7th most common cancer in men and 17th most common malignancy in women [1]. In 90-95\% of patients, BC is of urothelial origin [2], known as transitional cell carcinoma (TCC), and the rest small percentage is composed of non-urothelial BCs (NUBCs). Like TCC, these uncommon cancers exhibit geographical variations, e.g., regions with endemic schistosomal cystitis show a higher incidence of squamous cell carcinoma (SCC) [3]. Surgery can provide cure in localized cancers, whereas palliative chemotherapy or radiotherapy remain the mainstay of treatment in non-resectable and metastatic cancers [4]. These cancers can also present in combination with urothelial cancers as mixed histologies. All mixed or pure NUBCs are considered high-risk disease and generally have a poor prognosis as compare to patients with TCCs, and patients usually present at an advanced stage [5]. Due to these limitations in providing uniform treatment protocols for patients with NUBCs, a continuous research is required on this subject [6]. Here we share our experience in the treatment of these patients at a tertiary care referral cancer center in Pakistan.

\section{Materials And Methods}

Data of all patients presenting with BC to the department of Uro-oncology at Shaukat Khanum Memorial 


\section{Cureus}

Cancer Hospital and Research Center, Lahore, Pakistan, from January 2002 to July 2017 were reviewed retrospectively, and all adult patients between 18 and 87 years of age with histologically proven NUBCs were included in the study. Patients were followed for a median duration of 40 months (range: 5-92

months). Statistical analysis was conducted using IBM SPSS Statistics for Windows, Version 20.0 (IBM Corp., Armonk, NY, USA). The Kaplan-Meier method was used to estimate disease-free survival (DFS) and overall survival (OS). Formal approval was obtained from the Institutional Review Board before commencing data collection.

\section{Results}

A total of 20 patients were found to have NUBCs in the study duration. Out of them, 14 patients had adenocarcinoma, 3 had SCC, 3 had small cell carcinoma, and 1 had inflammatory myofibroblastic cancer. The median presenting age of the patients was 52 years (range: $34-87$ years), and hematuria was the commonest presenting concern, i.e., in 17 (85\%) patients. Three patients presented with lower urinary tract symptoms. Clinically, T3 was the commonest stage of presentation, and one patient had metastatic disease (Table 1).

\begin{tabular}{|c|c|c|c|c|c|}
\hline Variables & Stages & Adenocarcinoma, 14 (70.0\%) & SCC, 3 (15.0\%) & Small cell carcinoma, $2(10.0 \%)$ & IMT, 1 (5.0\%) \\
\hline \multirow{3}{*}{ CT } & T2 & $4(28.6)$ & - & - & - \\
\hline & T3 & $7(50.0)$ & $3(100.0)$ & - & $1(100.0)$ \\
\hline & T4 & $3(21.4)$ & - & $2(100.0)$ & - \\
\hline \multirow{2}{*}{$\mathrm{cN}$} & No & $11(78.6)$ & $3(100.0)$ & $1(50.0)$ & $0(0.0)$ \\
\hline & N1 & $3(21.4)$ & - & $1(50.0)$ & $1(100.0)$ \\
\hline \multirow{2}{*}{ cM } & M0 & $13(92.9)$ & $3(100.0)$ & $2(100.0)$ & $1(100.0)$ \\
\hline & M1 & $1(7.1)$ & - & - & - \\
\hline
\end{tabular}

\section{TABLE 1: Clinical disease staging}

SCC, squamous cell carcinoma; IMT, inflammatory myofibroblastic tumor; cT, clinical primary tumor; cN, clinical lymph nodes; cM, clinical metastasis; T2, tumor invades detrusor muscle; T3, tumor invades perivesical tissue; T4, tumor invades any of the following: prostate stroma, seminal vesicles, uterus, vagina, pelvic wall, abdominal wall; N0, no regional lymph node metastasis; N1, metastasis in a single lymph node in the true pelvis (hypogastric, obturator, external iliac, or presacral); M0, no distant metastasis; M1, distant metastases

The pathological staging according to tumor histologies is presented in Table 2. 


\section{Cureus}

\begin{tabular}{|c|c|c|c|c|c|}
\hline Variables & Stages & Adenocarcinoma, 14 (70.0\%) & SCC, $3(15.0 \%)$ & Small cell carcinoma, $2(10-.0 \%)$ & IMT, 1 (5.0\%) \\
\hline \multirow{5}{*}{ pT } & $\mathrm{Tx}$ & $2(14.3)$ & $1(33.3)$ & $2(100.0)$ & - \\
\hline & T1 & - & - & - & - \\
\hline & T2 & $6(42.9)$ & $1(33.3)$ & - & $1(100.0)$ \\
\hline & T3 & $4(28.6)$ & - & - & - \\
\hline & T4 & $2(14.3)$ & $1(33.3)$ & - & - \\
\hline \multirow{4}{*}{$\mathrm{pN}$} & $\mathrm{Nx}$ & $2(14.3)$ & $1(33.3)$ & $2(100.0)$ & - \\
\hline & No & $10(71.4)$ & $1(33.3)$ & - & $1(100.0)$ \\
\hline & N1 & $1(7.1)$ & $1(33.3)$ & - & - \\
\hline & N2 & $1(7.1)$ & - & & - \\
\hline
\end{tabular}

\section{TABLE 2: Pathological disease staging}

SCC, squamous cell carcinoma; IMT, inflammatory myofibroblastic tumor; pT, pathological primary tumor; pN, pathological primary lymph nodes; Tx, primary tumor cannot be assessed; T1, tumor invades subepithelial connective tissue; T2, tumor invades detrusor muscle; T3, tumor invades perivesical tissue; T4, tumor invades any of the following: prostate stroma, seminal vesicles, uterus, vagina, pelvic wall, abdominal wall; Nx, regional lymph nodes cannot be assessed; N0, no regional lymph node metastasis; N1, metastasis in a single lymph node in the true pelvis (hypogastric, obturator, external iliac, or presacral); N2, metastasis in multiple regional lymph nodes in the true pelvis (hypogastric, obturator, external iliac, or presacral)

Table 3 demonstrates the treatment patients received. It shows that patients with adenocarcinoma, as they presented at an earlier stage, could be offered curative surgical treatment in most instances. For patients with SCC, one out of three was able to undergo radical cystectomy with adjuvant radiotherapy cover, and the rest two could only be offered palliative chemotherapy. Patients with small cell carcinoma presented with $\mathrm{T} 4$ at the outset and received chemotherapy only.

\begin{tabular}{|c|c|c|c|c|c|}
\hline Variables & Categories & Adenocarcinoma, $n=14$ & SCC, $n=3$ & Small cell carcinoma, $n=2$ & IMT, $n=1$ \\
\hline \multirow{4}{*}{ Chemotherapy } & None & $11(78.5)$ & $1(33.3)$ & - & $1(100.0)$ \\
\hline & Adjuvant & 2 (14.3) & - & - & - \\
\hline & Definitive & - & - & & - \\
\hline & Palliative & $1(7.1)$ & $2(66.7)$ & $2(100.0)$ & - \\
\hline \multirow{4}{*}{ Radiotherapy } & None & 13 (92.9) & $2(66.7)$ & $2(100.0)$ & $1(100.0)$ \\
\hline & Adjuvant & - & $1(33.3)$ & - & - \\
\hline & Radical & - & - & - & - \\
\hline & Palliative & $1(7.1)$ & - & - & - \\
\hline \multirow{3}{*}{ Surgical procedure } & TURBT & $1(7.1)$ & $2(66.7)$ & $2(100.0)$ & - \\
\hline & Partial cystectomy & $9(64.3)$ & - & - & $1(100.0)$ \\
\hline & Radical cystectomy & $4(28.6)$ & $1(33.3)$ & - & - \\
\hline
\end{tabular}

\section{TABLE 3: Treatment modalities with respect to histology}

SCC, squamous cell carcinoma; IMT, inflammatory myofibroblastic tumor; TURBT, trans-urethral resection of bladder tumor

The median follow-up duration was 40 months (range: 5-92 months). During this period, 14 (70\%) patients were diagnosed with cancer recurrence or progression, with majority, i.e., 4 (20\%), exhibiting disease at multiple sites. Furthermore, all these patients died because of the disease progression itself (10 patients), and the remaining (4 patients) died of generalized ill health and comorbidities. On analysis, the mean DFS 


\section{Cureus}

was $59.10 \pm 8.66$ months (Figure 1 ), and the overall median survival was $56.0 \pm 8.94$ months (Figure 2). Furthermore, three- and five-year DFS was $60 \%$ and $51 \%$, respectively, and OS was $65 \%$ and $31 \%$, respectively.

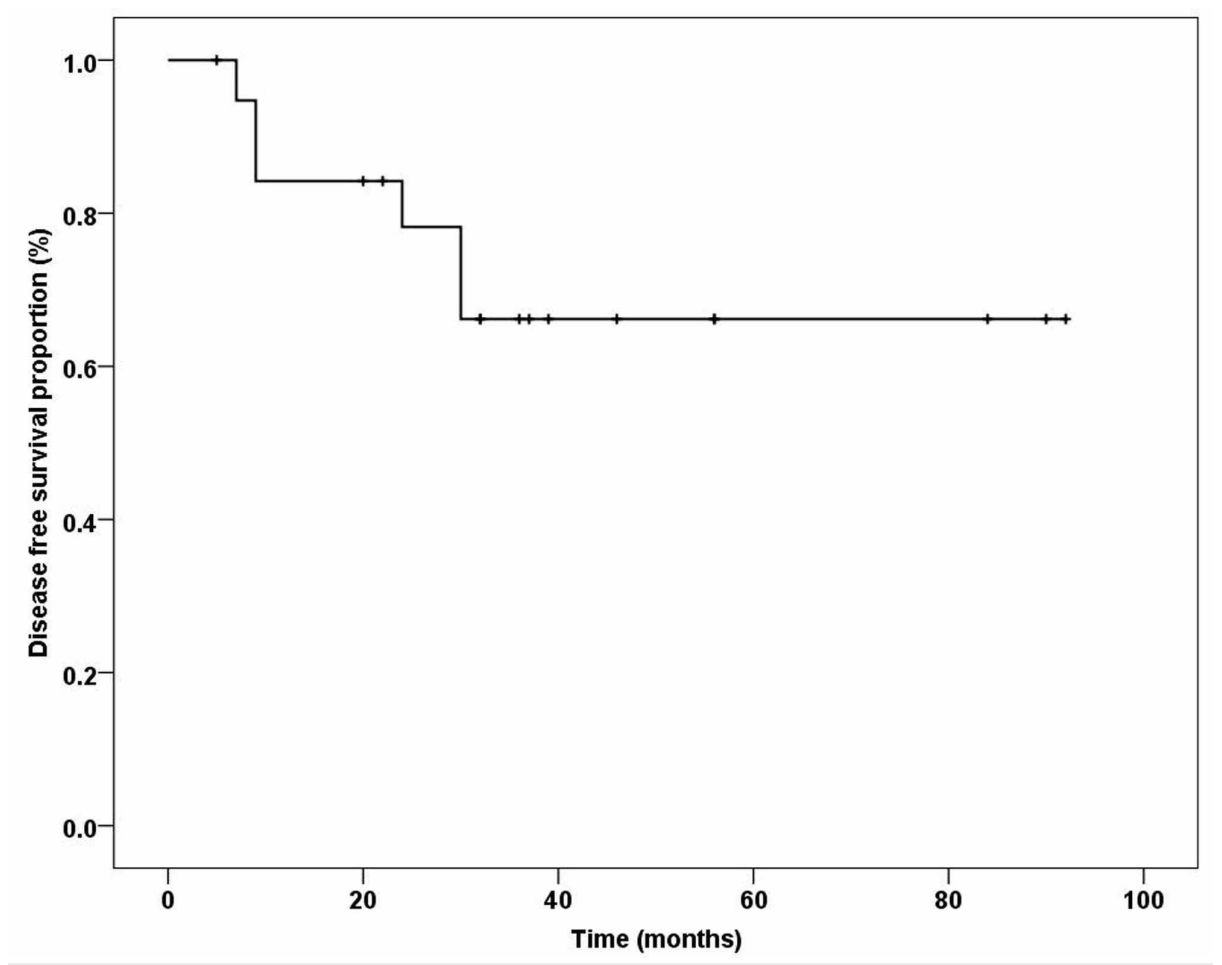

FIGURE 1: Disease-free survival for patients with non-urothelial bladder cancer $(n=20)$

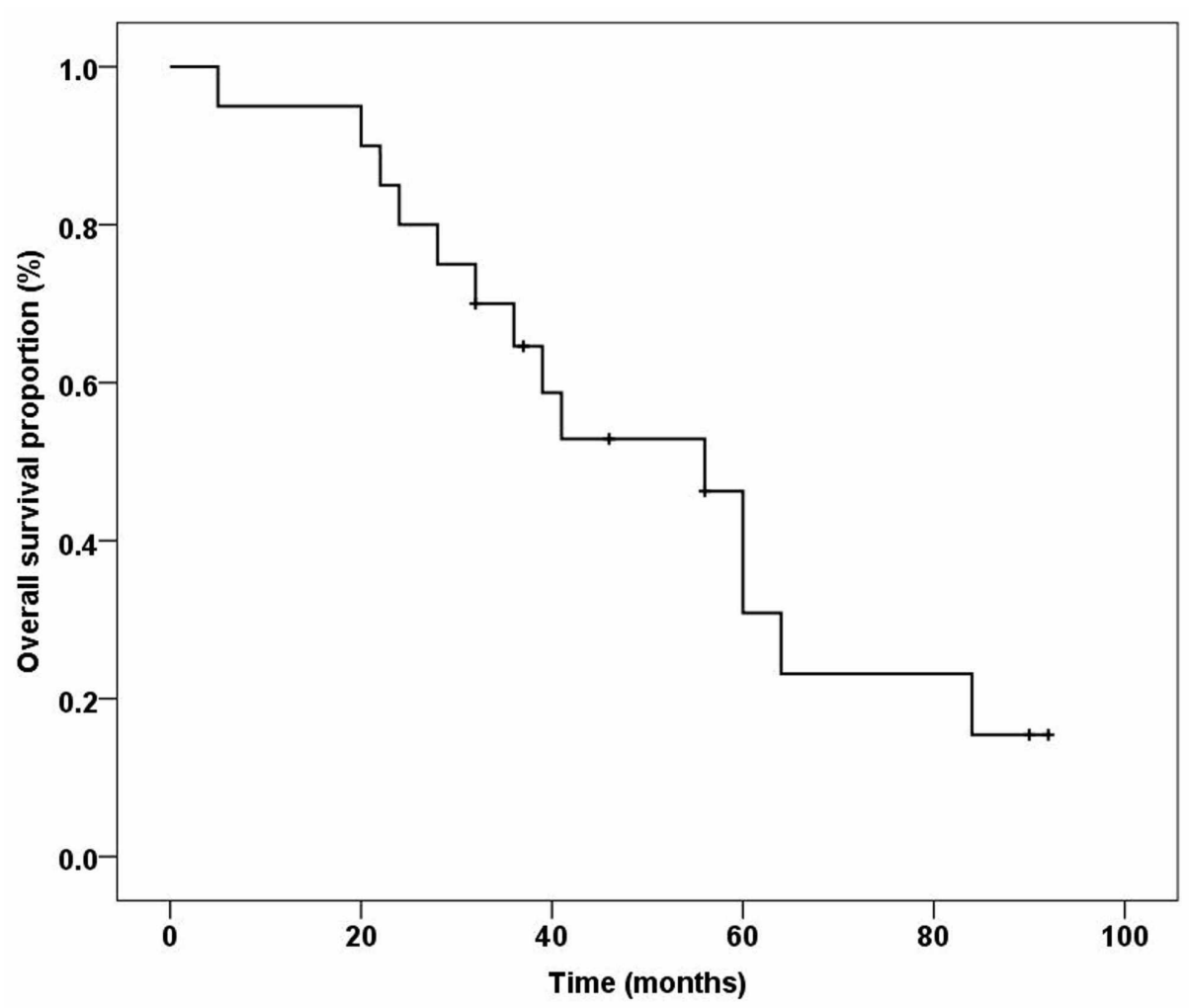

FIGURE 2: Overall survival with non-urothelial bladder cancer $(n=20)$ 


\section{Discussion}

NUBC is an aggressive disease, and early diagnosis and commencement of treatment are the main factors determining clinical outcomes [7]. Worldwide, SCC is reported to be the most common NUBC, accounting for 3-5\% of all BCs [8]. It is most common in Egypt and other African countries, where it is believed to be associated with chronic infection with Schistosoma haematobium [9]. Radical cystectomy is the treatment of choice for localized SCC because benefits from radiation are uncertain, and standard chemotherapy has little effect due to relative chemoresistance of disease [10]. Adenocarcinoma accounts for about $2 \%$ of BCs, and to establish the diagnosis of primary adenocarcinoma of the bladder, other possible primary tumors sites such as adenocarcinoma of the prostate or rectum should be ruled out [11,12]. Apart from endemic areas, adenocarcinoma is the most prevalent NUBC. Adenocarcinoma accounted for a major share of NUBC in our study. Standard treatment of all surgically resectable vesical adenocarcinomas consists of radical cystectomy and pelvic node dissection [13].

Small cell carcinoma is much rarer, accounting for less than $1 \%$ cases. It occurs mostly during the seventh and eight decades of life. Small cell carcinoma patients with localized disease should be managed radical cystectomy or multimodal therapy including surgery along with chemoradiation. Palliative chemotherapy is reserved for unresectable disease [14]. It carries poor prognosis due to its rarity and aggressive nature [15]. Accordingly, the two patients in our study presented with locally advanced unresectable disease at the outset and received palliative chemotherapy. Both of them died due to disease progression at 20 and 34 months follow-up. One of our patients was proven to have inflammatory myofibroblastic tumor. It is a rare NUBC of the bladder with an unknown possibility of malignant conversion [16]. Treatment strategy comprises transurethral resection, cystectomy, and radiotherapy. Our patient presented at the age of 34 years, was treated with partial cystectomy, and was on regular follow-up for the last 46 months. Additionally, in this study, three- and five-year OS of adenocarcinoma of the bladder was $63 \%$ and $27 \%$, respectively. Three-year OS of SCC and adenocarcinoma was $44.8 \%$ and 58.7\%, respectively [17]. In another study, five-year survival for SCC and adenocarcinoma was reported as $37 \%$ and 58\%, respectively [16]. The difference in OS at five years of adenocarcinoma was because patients presented with advanced disease stage and relatively small number of sample size as compared to published data.

The median survival of small cell cancer has been reported to be between 10 and 20 months, with 5-20\% 5year OS [18]. The OS of patients diagnosed with SCC and small cell carcinoma cannot be commented due to the small number of participants in our data. Standardized and further collaborative research is needed to move this field forward. Our data also highlight that tools are needed to detect this type of tumors early so that appropriate treatment can be commenced promptly.

\section{Conclusions}

NUBC is a rare disease entity that encompasses a variety of histological subtypes. Our data demonstrate that NUBC is an aggressive disease, which presents with advanced stage and has a poor prognosis. Treatment protocols are not uniform. A combined effort is needed among institutions, both nationally and internationally, to further elucidate the understanding of biology of these tumors and evaluate treatment protocols, which will help in improving long-term survival outcomes.

\section{Additional Information \\ Disclosures}

Human subjects: Consent was obtained by all participants in this study. Animal subjects: All authors have confirmed that this study did not involve animal subjects or tissue. Conflicts of interest: In compliance with the ICMJE uniform disclosure form, all authors declare the following: Payment/services info: All authors have declared that no financial support was received from any organization for the submitted work. Financial relationships: All authors have declared that they have no financial relationships at present or within the previous three years with any organizations that might have an interest in the submitted work. Other relationships: All authors have declared that there are no other relationships or activities that could appear to have influenced the submitted work.

\section{References}

1. Antoni S, Ferlay J, Soerjomataram I, Znaor A, Jemal A, Bray F: Bladder cancer incidence and mortality: a global overview and recent trends. Eur Urol. 2017, 71:96-108. 10.1016/j.eururo.2016.06.010

2. Parkin DM, Bray F, Ferlay J, Pisani P: Global cancer statistics, 2002. CA Cancer J Clin. 2005, 55:74-108. 10.3322/canjclin.55.2.74

3. Maia MC, Hansen A, Alves C, Salah S: Biomarkers in non-Schistosomiasis-related squamous cell carcinoma of the urinary bladder: a review. Crit Rev Oncol Hematol. 2019, 135:76-84. 10.1016/j.critrevonc.2019.01.008

4. Miyazaki J, Nishiyama H: Epidemiology of urothelial carcinoma. Int J Urol. 2017, 24:730-734. 10.1111/iju.13376

5. Cai T, Tiscione D, Verze P, et al.: Concordance and clinical significance of uncommon variants of bladder urothelial carcinoma in transurethral resection and radical cystectomy specimens. Urology. 2014, 84:11411146. 10.1016/j.urology.2014.06.032

6. Aragon-Ching J, Trump D, Wang H: Comparative analyses of trends and survival in patients with urothelial 
versus nonurothelial bladder carcinoma: National Cancer Database (NCDB) analysis. J Clin Oncol. 2019, 37:402-402. 10.1200/JCO.2019.37.7_suppl.402

7. Patel S, Weiner A, Keegan K, Morgan T: Oncologic outcomes in patients with nonurothelial bladder cancer . Indian J Urol. 2018, 34:39-44. 10.4103/iju.IJU_115_17

8. Abol-Enein H, Kava BR, Carmack AJ: Nonurothelial cancer of the bladder. Urology. 2007, 69:93-104. 10.1016/j.urology.2006.08.1107

9. Kassouf W, Spiess PE, Siefker-Radtke A, et al.: Outcome and patterns of recurrence of nonbilharzial pure squamous cell carcinoma of the bladder: a contemporary review of The University of Texas MD Anderson Cancer Center experience. Cancer. 2007, 15:764-769. 10.1002/cncr.22853

10. Swanson DA, Liles A, Zagars GK: Preoperative irradiation and radical cystectomy for stages T2 and T3 squamous cell carcinoma of the bladder. J Urol. 1990, 143:37-40. 10.1016/S0022-5347(17)39857-9

11. Wilson TG, Pritchett TR, Lieskovsky G, Warner NE, Skinner DG: Primary adenocarcinoma of bladder . Urology. 1991, 38:223-226. 10.1016/S0090-4295(91)80349-C

12. Ravi K, Kumar T, Bakshi H, Desai J, Sen S, Yadav V: Non urothelial bladder cancers: a case series . Indian J Surg Oncol. 2013, 4:2-8. 10.1007/s13193-012-0176-9

13. Siefker-Radtke AO, Gee J, Shen YU, Wen S, Daliani D, Millikan RE, Pisters LL: Multimodality management of urachal carcinoma: the MD Anderson Cancer Center experience. J Urol. 2003, 169:1295-1298. 10.1097/01.ju.0000054646.49381.01

14. Lohrisch C, Murray N, Pickles T, Sullivan L: Small cell carcinoma of the bladder: long term outcome with integrated chemoradiation. Cancer. 1999, 86:2346-2352. 10.1002/(sici)10970142(19991201)86:11<2346::aid-cncr24>3.0.c0;2-5

15. Dong WX, Ping YX, Liang WC, Jian LZ, Lin ZJ: Small cell carcinoma of the urinary bladder diverticulum: a case report and review of the literature. J Cancer Res Ther. 2013, 9:151-153. 10.4103/0973-1482.110372

16. Aragon-Ching J, Henson D: Differences in survival among non-urothelial bladder cancers: analyses of SEER 1988-2008. J Clin Oncol. 2018, 36:425-425. 10.1200/JCO.2018.36.6_suppl.425

17. Kamat A, Hahn N, Efstathiou J, et al.: Bladder cancer. Lancet. 2016, 388:2796-2810. 10.1016/S01406736(16)30512-8

18. Lynch SP, Shen Y, Kamat A, et al.: Neoadjuvant chemotherapy in small cell urothelial cancer improves pathologic downstaging and long-term outcomes: results from a retrospective study at the MD Anderson Cancer Center. Eur Urol. 2013, 64:307-313. 10.1016/j.eururo.2012.04.020 